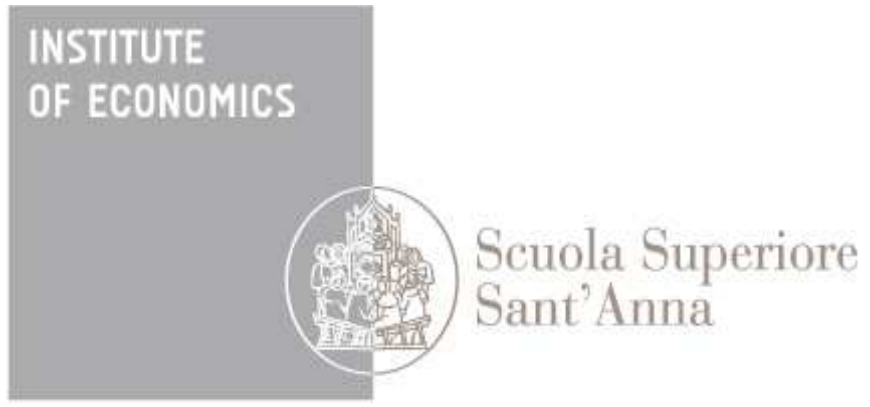

LEM | Laboratory of Economics and Management

Institute of Economics

Scuola Superiore Sant'Anna

Piazza Martiri della Libertà, 33 - 56127 Pisa, Italy ph. +3905088.33 .43$

institute.economics@sssup.it

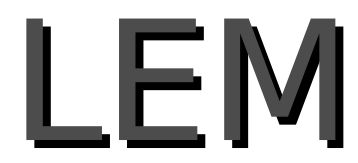

Working Paper Series

\title{
Rationality and Asset Prices under Belief Heterogeneity
}

Daniele Giachini ${ }^{\circ}$

'Institute of Economics, Scuola Sant'Anna, Pisa, Italy 


\title{
Rationality and Asset Prices under Belief Heterogeneity
}

\author{
Daniele Giachini \\ Istituto di Economia, Scuola Superiore Sant'Anna \\ Piazza Martiri della Libertà 33, 56127 Pisa - Italy
}

February 21, 2018

\begin{abstract}
In this paper I study the relationship between rationality and asset prices when agents have heterogeneous and incorrect beliefs about future events. Using the fully rational pricing as a benchmark, I show that when agents behave according to the Subjective Generalized Kelly rule (Bottazzi et al., 2017), which is not optimal under agents' beliefs, the long-run pricing performance is at least as good as the one emerging from an economy where agents maximize their preferences under rational price expectations. Indeed, there exist generic cases in which expected long-run prices of the Subjective Generalized Kelly economy approximate better the rational pricing than those attained by the utility maximizers economy. Moreover in the limit of agents having a discount factor equal to one the prices of the Subjective Generalized Kelly economy converge to those of the fully rational economy. Hence the fact that agents use non-optimal (heuristic) decision rules may correct for biases in beliefs and, as a consequence, improve the pricing performance of the economy.
\end{abstract}

JEL Classification: C60, D53, D81, G11, G12

Keywords: Belief Heterogeneity, Rationality, Investment Rules, Heuristics, Financial Markets, Asset Pricing. 


\section{Introduction}

Standard models of asset pricing, like the one of Lucas (1978), assume that agents are fully rational. This basically means that, on the one hand, agents know every detail of the economy, while, on the other, their behavior derives from (or is consistent with) utility maximization. Thus, among the characteristics of the economy agents know, there is the probability distribution according to which future events occur: a fully rational agent can exactly evaluate the likelihood of every possible future state of the economy. This turns out to be a very strong and rather unrealistic assumption, especially after the 2007-2008 financial crisis. Hence, understanding what happens to asset prices when no one knows the true distribution becomes a relevant issue. Blume and Easley (2009) address it keeping the assumption that agents behave as rationally as they can under their heterogeneous and, possibly, incorrect beliefs. They show how in the long-run market prices generically reflect the beliefs of the most accurate agent. ${ }^{1}$ This sounds as a second-best: when no one knows exactly the true distribution, prices are at least able to reveal the best evaluation among those of market participants. Moreover, the standard way in which economists address bounded rationality would suggest that if we move further away from full rationality, e.g. assuming that agents' behavior is not consistent with utility maximization but relies on heuristic rules, pricing will only get worst. ${ }^{2}$

On the link between heuristic rules and performances the work of Gigerenzer and Brighton (2009) supports a different view: in complex environments using a simple "fast-and-frugal" decision rule improves outcomes. Thus, could it be that moving further away from full rationality actually improves pricing instead of worsening it? To investigate that I compare the pricing performance of different economies ranked by rationality. In particular I use as benchmark the prices one gets in a long-lived asset pure exchange economy populated by expected utility maximizers with fully rational expectations (henceforth FR economy) and I compute the long-run pricing in two long-lived asset economies where agents have heterogeneous and incorrect beliefs about the likelihood of states of the world to appear. In one economy agents maximize a standard expected utility function under rational price expectations ${ }^{3}$ (henceforth EU economy) while in the other

\footnotetext{
${ }^{1}$ This is always true in the setting of Blume and Easley (2009) when agents share the same discount factor. See Section 2 for further details.

${ }^{2}$ See section 2 for more details.

${ }^{3}$ With rational price expectations I mean that agents are able to compute the price of every asset in every possible future state of the world. To do that they should know every detail of the economy but the true probability according to which future states appear. Thus each agent knows the beliefs of the others and assuming belief heterogeneity means that they agree to disagree.
} 
agents behave according to the Subjective Generalized Kelly rule ${ }^{4}$ introduced in Bottazzi et al. (2017) (henceforth SGK economy). Since the EU economy I shall consider is a special case of the one studied by Blume and Easley (2009), my analysis focuses on SGK long-run pricing and shows how, under heterogeneous and biased beliefs, in the long-run the normalized prices of a SGK economy are either the same of the EU economy or they are such that their expectation approximates better the FR pricing than the EU normalized prices. Moreover, if agents are patient enough (that is, they have a discount factor close to 1) then SGK economy's prices converge to those of the FR economy. Hence it turns out that, in economies where no one can evaluate correctly the likelihood of future events, having agents that behave using simple decision rules may improve the overall performance of the system. To understand that notice how using non-optimal rules may correct for the inaccuracy of agents' probabilistic evaluations: a simple decision rule implemented under biased beliefs may map into an optimal rule implemented under rather sophisticated and accurate beliefs (Bottazzi et al., 2017). Thus an agent who assigns probabilities to future events in an incorrect manner may behave as if she could make more accurate likelihood evaluations simply following an heuristic decision rule. This enhancement in decision making is incorporated in prices, improving the asset pricing performance of the economy.

The structure of the paper is the following: section 2 reviews the most relevant literature, section 3 introduces the model and the different types of agents' behavior, section 4 develops the analysis and discusses the results, section 5 concludes.

\section{Related Literature}

The analysis pursued here is mainly related to two steams of literature: the one investigating individual rationality and aggregate outcomes, and the one studying market selection and pricing.

Contributions on the link between individual rationality and aggregate outcomes are summarized and discussed by Fehr and Tyran (2005). The two authors argue how many economists, even recognizing the presence of less than fully rational agents in the market, believe that market interaction corrects "anomalous behavior". They report five common hypotheses about how this correction should happen. The first one assumes random deviation from full rationality which cancel out during aggregation. The second one considers learning: irrational agents learn from their mistakes and over time they become rational. The third is connected to the results of Gode and Sunder (1993) about double action markets with zero-

\footnotetext{
${ }^{4}$ Such rule consists in saving in each period a share of wealth equal to the discount factor and investing in every asset a fraction of the saved wealth proportional to the asset's expected dividend computed using subjective probabilities.
} 
intelligence traders and states that market structure itself drives toward rational outcomes. The fourth is a market selection argument, which recalls Alchian (1950) and Friedman (1953): rational agents make the highest profits and, thus, drive irrational individuals out from the market. The last argument states that, in market characterized by a large number of agents, irrational individuals hold extreme position while rational ones are marginal buyers or sellers, thus in equilibrium only rational agents matter. Fehr and Tyran note, however, that there exist several cases in which those mechanisms do not work and conclude that what really determines aggregate outcomes is the fact that agents' strategies are complementary or substitutes. In the former case a small amount of individual irrationality may drive aggregate outcomes far away from rational values. In the latter case, instead, the presence of few rational agents is enough to ensure the convergence to rational outcomes. Thus the link between individual rationality and aggregate outcomes the two authors depict is clear: a lack of the first is a potential harm for the second.

The idea that agents' bounded rationality explains deviations from rational outcomes underlies the whole field of behavioral economics (Camerer et al., 2011). Such literature builds upon the work of Tversky and Kahneman (1974) which addresses cognitive biases stemming from judgmental heuristics. The fundamental point is that widespread heuristics affect decision making in a detrimental way since they let agents make non-optimal choices. This view is in part challenged by the work of Gigerenzer et al. (1999), Gigerenzer and Brighton (2009) and Gigerenzer and Gaissmaier (2011). They build upon the seminal paper of Simon (1955) which introduced the idea that, because of computational and informational limitations, agents are not able to optimize their choices. According to Simon economic agents simplify the decision problem using procedures that lead to a satisfactory choice. Gigerenzer and coauthors show how such procedures are indeed heuristics that actually improve decision making. The contrast between the contributions of Tversky and Kahneman and the work of Gigerenzer and coauthors is that the latter considers individuals embedded in a complex environment where designing an optimal choice is not conceivable. Hence heuristics became simple tricks to get the most out of a situation which is uncertain in the sense of Knight (1921). Moreover the authors also show how in many cases trying to increase the rationality of a choice (e.g. gathering more information) worsens performances because of a variance-bias trade-off. This idea is investigated by Dosi et al. (2017) in the context of a macroeconomic agent based model in which the aggregate performance of the economy is evaluated under different expectation formation rules. They show how, in a complex evolving environment, the fact that agents use sophisticated expectation formation rules worsens both the individual performance and the aggregate outcome of the economy with respect to the case in which they adopt simple myopic expectations. Thus they conclude that, under 
the Knightian uncertainty introduced by technical change, interaction, and imperfect information, relying on simple heuristics solves the variance-bias trade-off and improves aggregate performances. A similar point emerges from the analysis of Kirman (2010): the interaction of unsophisticated heterogeneous individuals, like buyers in fish markets (Härdle and Kirman, 1995; Kirman and Vriend, 2000; Gallegati et al., 2011) or even ants (Kirman, 1993), often creates aggregate outcomes that are well-behaved while the interaction of rather rational agents may generate outcomes that are far away from rational levels.

Regarding selection and pricing, it is formally investigated by Blume and Easley (2009) considering an exchange economy with complete markets, constant endowment, and agents that maximize the expectation of their geometrically discounted utility of consumption over an infinite horizon. Taking into account an agent specific survival index that involves discount factor and belief accuracy, they show how long-run prices generically reflect the beliefs of the agent with the highest survival index, i.e. the one markets select for. They also note how there exist hairline cases in which at least two agents manage to survive (same maximal survival index) and this implies that prices fluctuate in-between the beliefs of those traders. ${ }^{5}$ Dindo and Massari (2017) show how such cases become generic when agents correct their beliefs with equilibrium prices. Moreover in those situations risk neutral probabilities implied by market prices turn out to be more accurate than the beliefs of any agent. They also show such accuracy becomes higher as agents increase the correction applied and, in the limit of agents using as beliefs equilibrium prices, risk neutral probabilities reveal the true probabilities of the underlying process. Massari (2017a), instead, studies selection and pricing in large economies (i.e. economies populated by a continuum of traders), finding that efficient prices emerge even when accurate traders vanish because of the selection forces operating in the market. At the same time, as Kogan et al. (2006), Cvitanić et al. (2012), and Kogan et al. (2017) argue, vanishing agents may have an impact on long-run prices in economies without intermediate consumption and populated by agents whose objective function depends on their wealth at a finite horizon, or in economies where agents' risk aversion is unbounded.

While papers in the previous paragraph build upon the standard utility maximization framework imposing general equilibrium, other contribution focus on selection and pricing when agents use behavioral rules. Many of them consider heterogeneous agent models $(\mathrm{HAMs})^{6}$ whose main feature is the interaction of

\footnotetext{
${ }^{5}$ Jouini and Napp (2011) provide an account of the differences between economies characterized by such unbiased disagreement and standard rational settings.

${ }^{6}$ See e.g. Day and Huang (1990); Kirman (1991); Lux (1995); Sethi (1996); Arthur et al. (1997); Brock and Hommes (1997, 1998); Franke and Sethi (1998); Bullard and Duffy (1999); Lux and Marchesi (1999); Chiarella and He (2001); Chiarella et al. (2002); Iori (2002); Chiarella
} 
heterogeneous and boundedly rational agents. Such class of models proved able to generate price (or return) dynamics that resemble those recorded empirically or experimentally by means of selection processes among different behaviors. Using a modeling framework closer to the one I consider, Kets et al. (2014), Bottazzi and Giachini (2016), and Bottazzi and Giachini (2017) investigate market selection and bet pricing in prediction markets. Kets et al. (2014) show by means of numerical simulations that, in the case of Fractional Kelly betting, situations in which the average price matches the true underlying probability arise. Those cases are driven by the long-run survival of agents with heterogeneous beliefs. They also conjecture how in the limit of agents investing according to prices, the expected bet price reveals the probability of observing the event. Bottazzi and Giachini (2017) consider a model equivalent to the one of Kets et al. (2014) with Fractional Kelly traders and provide an analytical method to approximate the long-run invariant distributions of wealth and prices. They use it to prove the conjecture of Kets et al. (2014). Bottazzi and Giachini (2016) build upon Bottazzi and Giachini (2017) and prove that there exists generic cases in which the expected price emerging from a prediction market can be far away from the truth. In the end, Bottazzi et al. (2018) study return dynamics in a SGK economy similar to the one considered here and show how short-term mometum and long-term reversal generically occur because of the wealth redistribution process taking place under persistent heterogeneity.

\section{Model}

Consider a pure exchange economy in discrete time indexed by $t=0,1, \ldots$ with a homogeneous consumption good. In the model there are two agents (indexed as $i \in\{1,2\}$ ) who exchange two long-lived assets (indexed by $k \in\{1,2\}$ ). I assume that in $t=0$ each agent $i$ has a certain amount of initial endowment $W_{0}^{i}$ (in terms of the homogeneous good) which is used to purchase the assets and to support time $t=0$ consumption. In every date $t>0$ the aggregate endowment is $Y$ and one of the two possible states of the world occurs: in the first state the first asset pays the aggregate endowment as dividend and the second pays nothing, while in the second state of the world it is the other way round. Uncertainty, entering the model from date $t=1$ onwards, is modeled in terms of a Bernoulli process $s_{t} \in\{1,0\}$ where the first state is the realization $s_{t}=1$ and the second state is the realization $s_{t}=0$. The probability of observing the first state at a certain date $t>0$ is $\pi^{*}$ such that the probability of observing the second state is $1-\pi^{*}$.

et al. (2006); Alfarano et al. (2008); Chiarella et al. (2009); Anufriev and Bottazzi (2010); Anufriev and Dindo (2010); Tedeschi et al. (2012); Anufriev and Hommes (2012). See Hommes (2006) for a survey of HAMs in economics and finance. 
Following the notation of Blume and Easley (2009), I call $\Sigma$ the set of all the sequences of states whose generic element is $\sigma=\left(s_{1}, s_{2}, \ldots\right)$. This is usually called a path and when I need to indicate its partial history at date $t$, I use $\sigma^{t}$. I denote as $\mathbb{P}$ the probability measure on $\Sigma$ whose distribution is consistent with the Bernoulli draws introduced in advance. Expectations are denoted as E and when subscripts or superscripts are missing it is taken with respect to $\mathbb{P}$. $\mathcal{F}$ is the sigma-field on $\Sigma$ such that $(\Sigma, \mathcal{F}, \mathbb{P})$ is a well defined measurable space on which everything is build. The variables I am going to introduce are of the form $x_{t}$ which means they are $\mathcal{F}_{t}$-measurable, where $\mathcal{F}_{t}$ is the date $t$ sigma-field.

Next I assume that dividends are paid at the beginning of the period, assets are in unitary supply and prices are fixed in every period via market clearing conditions. Defining the dividend of asset $k$ in $t$ as $D_{k, t}$, the price of asset $k$ in $t$ as $P_{k, t}$, the consumption of agent $i$ in $t$ as $C_{t}^{i}$, the holding of asset $k$ in $t$ by agent $i$ as $h_{k, t}^{i}$, one has that agent $i$ budget constraint in $t=0$ is

$$
C_{0}^{i}=W_{0}^{i}-\sum_{k=1}^{2} P_{k, 0} h_{k, 0}
$$

while, for any $t>0$, it is

$$
C_{t}^{i}+\sum_{k=1}^{2} P_{k, t} h_{k, t}^{i}=\sum_{k=1}^{2}\left(D_{k, t}+P_{k, t}\right) h_{k, t-1}^{i} .
$$

Here I can define agent $i$ 's wealth in $t>0$ as her pre-consumption net worth, I call it $W_{t}^{i}$ and it reads

$$
W_{t}^{i}=\sum_{k=1}^{2}\left(D_{k, t}+P_{k, t}\right) h_{k, t-1}^{i} .
$$

Let me define the share of wealth that agent $i$ dedicates to investment in $t$ as $\delta_{t}^{i} \in$ $(0,1)$, such that her consumption is $C_{t}^{i}=\left(1-\delta_{t}^{i}\right) W_{t}^{i}$. Moreover I call $\alpha_{k, t}^{i} \in(0,1)$ the fraction of invested wealth that agent $i$ allocates to asset $k$ in $t$, obviously $\alpha_{1, t}^{i}+\alpha_{2, t}^{i}=1$. Notice that by definition one has $P_{k, t} h_{k, t}^{i}=\delta_{t}^{i} \alpha_{k, t}^{i} W_{t}^{i}$ such that agent $i$ 's holding of asset $k$ at in $t$ is

$$
h_{k, t}^{i}=\frac{\delta_{t}^{i} \alpha_{k, t}^{i} W_{t}^{i}}{P_{k, t}} .
$$

Since assets are in unitary supply, the market clearing condition of asset $k$ in $t$ is $1=h_{k, t}^{1}+h_{k, t}^{2}$ which implies by (4) that

$$
P_{k, t}=\sum_{i=1}^{2} \delta_{t}^{i} \alpha_{k, t}^{i} W_{t}^{i}
$$


Substituting (4) in (3) one recovers agent $i$ 's wealth dynamics

$$
W_{t}^{i}=\delta_{t-1}^{i} W_{t-1}^{i} \sum_{k=1}^{2} \frac{D_{k, t}+P_{k, t}}{P_{k, t-1}} \alpha_{k, t-1}^{i}
$$

\subsection{Agents' Behavior}

The model just introduced will be studied under three different specifications of agents' behavior such that I have three different economies ordered in terms of agents' rationality. First of all I assume that agents have a homogeneous discount factor $\beta$ and this is common to every economy I will consider. In the first economy I assume that agents are expected utility maximizers with fully rational expectation, that is, agent $i$ makes her consumption and investment decisions solving the following problem

$$
\max _{\delta_{t}^{i}, \alpha_{k, t}^{i} ; \forall k, t, \sigma} \mathrm{E}\left[\sum_{t=0}^{\infty} \beta^{t} U\left(C_{t}^{i}\right)\right] \quad \text { s.t. budget constraints (1), (2), }
$$

where $U(\cdot)$ is a utility function (homogeneous across agents ${ }^{7}$ ) which is $\mathcal{C}^{1}$, strictly concave, strictly monotonic, and satisfies the Inada condition at $0 .^{8}$

In the second economy agents are expected utility maximizers with rational price expectations and beliefs $\pi^{1}, \pi^{2}$. Agents' beliefs are constant, biased, and heterogeneous, moreover, without loss of generality, I call agent 1 the one who assigns a larger probability to the occurrence of $s_{t}=1$. In formal terms I assume the following

A1 $\pi^{1}, \pi^{2} \in \mathbb{R}$ with $\pi^{1} \neq \pi^{*}, \pi^{2} \neq \pi^{*}$ and $0<\pi^{2}<\pi^{1}<1$.

Thus agent $i$ solves the problem

$$
\max _{\delta_{t}^{i}, \alpha_{k, t}^{i} ; \forall k, t, \sigma} \mathrm{E}^{i}\left[\sum_{t=0}^{\infty} \beta^{t} U\left(C_{t}^{i}\right)\right] \quad \text { s.t. budget constraints (1), (2), }
$$

where $\mathrm{E}^{i}$ must be intended as the expectation computed according to agent $i$ 's beliefs and $U(\cdot)$ is a utility function as in advance. Under these assumptions the second economy is just a special case of the one studied in Blume and Easley (2009).

\footnotetext{
${ }^{7}$ Since the aggregate endowment is constant across states, heterogeneous risk preferences do not affect the results. However, to keep consistency with the fact that agents in the SGK economy share the same behavioral rule, I assume homogeneous preferences both in the FR economy and in the EU economy.

${ }^{8}$ That is, it respects assumption A1 of Blume and Easley (2009).
} 
In the last economy agents have the same heterogeneous and incorrect beliefs $\pi^{1}, \pi^{2}$ as in the second economy while consume and invest according to the Subjective Generalized Kelly rule introduced in Bottazzi et al. (2017). The Subjective Generalized Kelly rule consists in consuming in every period a fraction $1-\beta$ of wealth and investing the remaining in the two assets proportionally to the next period expected dividend. ${ }^{9}$ That is, in every $t \in \mathbb{N}_{0}$ for every agent $i=1,2$ one has

$$
\delta_{t}^{i}=\beta, \quad \alpha_{1, t}^{i}=\pi^{i}, \quad \alpha_{2, t}^{i}=1-\pi^{i} .
$$

Notice that under these assumptions the third economy is just a special case of the one analyzed in Bottazzi et al. (2017).

\section{Selection and Pricing}

In the economies I have introduced pricing is deeply related to market selection dynamics. Thus, before going on, I need: $i$ ) to define what I mean by survival, dominance and vanishing; ii) to introduce a notion of accuracy; iii) to define a function which will be used to assess long-run selection in Subjective Generalized Kelly traders economies. Calling $w_{t}^{i}=W_{t}^{i} /\left(W_{t}^{1}+W_{t}^{2}\right)$ the normalized wealth of agent $i$ at time $t$, the following Definition formalizes long-run outcomes.

Definition 4.1. Agent $i$ dominates if

$$
\lim _{t \rightarrow \infty} w_{t}^{i}=1 \quad \text { a.s }
$$

Agent $i$ survives if

$$
\limsup _{t \rightarrow \infty} w_{t}^{i}>0 \quad \text { a.s . }
$$

If an agent does not survive then she vanishes.

Following Blume and Easley (2009) and Dindo and Massari (2017) I use KullbackLeibler divergence (or relative entropy) to measure accuracy of probability distributions.

Definition 4.2. Consider $(\pi, 1-\pi),\left(\pi^{\prime}, 1-\pi^{\prime}\right)$, and $\left(\pi^{*}, 1-\pi^{*}\right)$ with $\pi, \pi^{\prime}, \pi^{*} \in \mathbb{R}$ and $0<\pi<1,0<\pi^{\prime}<1,0<\pi^{*}<1$. I say that $(\pi, 1-\pi)$ is more accurate than

\footnotetext{
${ }^{9}$ Such rule is a fixed-mix investing strategy coupled with constant saving. Bottazzi et al. (2017) show that it corresponds to the optimal behavior of an expected utility maximizer with logarithmic preferences when the agent has all the wealth in the economy, otherwise it is not optimal under the agent's beliefs. Notice also that, in the framework studied here, it matches the prescription "bet your beliefs" of Breiman (1961).
} 
$\left(\pi^{\prime}, 1-\pi^{\prime}\right)$, or $(\pi, 1-\pi)$ approximates better $\left(\pi^{*}, 1-\pi^{*}\right)$ than $\left(\pi^{\prime}, 1-\pi^{\prime}\right)$, if its Kullback-Leibler divergence with respect to $\left(\pi^{*}, 1-\pi^{*}\right)$, defined as

$$
K L\left(\pi^{*} \| \pi\right)=\pi^{*} \log \frac{\pi^{*}}{\pi}+\left(1-\pi^{*}\right) \log \frac{1-\pi^{*}}{1-\pi},
$$

is smaller than the Kullback-Leibler divergence of $\left(\pi^{\prime}, 1-\pi^{\prime}\right)$ with respect to $\left(\pi^{*}, 1-\right.$ $\left.\pi^{*}\right)$. That is

$$
K L\left(\pi^{*} \| \pi\right)-K L\left(\pi^{*}|| \pi^{\prime}\right)<0
$$

To avoid hairline cases I assume that beliefs are such that their Kullback-Leibler divergence with respect to the truth is not equal.

A2 Given $\pi^{*}, \pi^{1}$ and $\pi^{2}$ are such that $K L\left(\pi^{*} \| \pi^{1}\right) \neq K L\left(\pi^{*} \| \pi^{2}\right)$.

The last ingredient I need is the function that Bottazzi et al. (2017) use to assess long-run selection outcomes in Subjective Generalized Kelly traders economies. Using generic beliefs $\pi, \pi^{\prime} \in \mathbb{R}$ with $0<\pi<1$ and $0<\pi^{\prime}<1$, it reads

$$
\mu\left(\pi^{\prime}, \pi\right)=\pi^{*} \log \frac{\beta \pi+(1-\beta) \pi^{\prime}}{\pi}+\left(1-\pi^{*}\right) \log \frac{\beta(1-\pi)+(1-\beta)\left(1-\pi^{\prime}\right)}{1-\pi} .
$$

Even here possible hairline cases may arise, thus I assume that beliefs are such that $\mu(\cdot, \cdot)$ has a definite sign.

A3 Given $\pi^{*}$ and $\beta, \pi^{1}$ and $\pi^{2}$ are such that $\mu\left(\pi^{1}, \pi^{2}\right) \neq 0$ and $\mu\left(\pi^{2}, \pi^{1}\right) \neq 0$.

To avoid confusion in what follows I will use the superscript $F R$ for the variables of the FR economy (like consumption, prices and wealth) while I will use the superscript $E U$ for the variables of the EU economy. Instead I will not use any superscript for the variables of the SGK economy in order to improve readability and notation.

\subsection{Pricing in FR economies}

From agent $i$ 's first order conditions one can derive the following formula for asset pricing which is similar to the one derived in Lucas (1978):

$$
P_{k, t}^{F R}=\mathrm{E}_{t}\left[\beta \frac{U^{\prime}\left(C_{t+1}^{F R, i}\right)}{U^{\prime}\left(C_{t}^{F R, i}\right)}\left(P_{k, t+1}^{F R}+D_{k, t+1}\right)\right], \quad k=1,2, t>0,
$$

where $\mathrm{E}_{t}$ means that the expectation is conditional on the information available at time $t$ and $U^{\prime}(\cdot)$ is the marginal utility. In this economy agents share the same 
beliefs and discount factor, thus, given the aggregate endowment equal to $Y$ in every $t>0$ and for any state of the world, one has that the consumption of each agent in every $t>0$ is constant. Simplifying and substituting one gets

$$
P_{k, t}^{F R}=\mathrm{E}_{t}\left[\sum_{\tau=1}^{\infty} \beta^{\tau} D_{k, t+\tau}\right], \quad k=1,2, t>0 .
$$

Then, considering the i.i.d. nature of the underlying stochastic process, under general equilibrium one has

$$
P_{1, t}^{F R}=\pi^{*} Y \frac{\beta}{1-\beta}, \quad P_{2, t}^{F R}=\left(1-\pi^{*}\right) Y \frac{\beta}{1-\beta}, \quad \forall t>0 .
$$

Dividing by the sum of prices one gets the normalized prices of asset $1\left(p_{t}^{F R}\right)$ and asset $2\left(1-p_{t}^{F R}\right)$. Focusing on asset 1 it is $p_{t}^{F R}=\pi^{*}$, which means that, under rational expectations, in every $t>0$ prices reveal the probability structure governing the underlying stochastic process.

\subsection{Pricing in EU Economies}

Since this economy is just a special case of those studied in Blume and Easley (2009), long-run pricing follows from a straightforward application of their results. As stated in advance, the authors show that two cases are possible in the longrun. Under discount factor homogeneity, if one agent has the most accurate beliefs then normalized prices converge to her beliefs. If there are more than one agent with equally accurate but heterogeneous beliefs, then prices fluctuate in-between their evaluations. However, notice that assumption A2 excludes the possibility of having more than one agent with equally accurate beliefs. Thus in the EU economy studied here long-run normalized prices show the most accurate beliefs in the market. Calling $p_{t}^{E U}$ the normalized price of asset 1 at time $t$, I report the results of Blume and Easley (2009) adapted to the model analyzed here in the following Proposition.

Proposition 4.1 (Blume-Easley). In the EU economy defined in advance, under assumptions A1 - A2 and calling agent $i$ the one with the most accurate beliefs, in general equilibrium one has almost surely

$$
\lim _{t \rightarrow \infty} p_{t}^{E U}=\pi^{i}
$$

Proof. See Appendix A. 


\subsection{Pricing in SGK Economies}

The analysis of long-run pricing in SGK economies pursued in this Section builds upon the results of Bottazzi et al. (2017). The first important result that Bottazzi et al. (2017) prove is that prices and returns in a SGK economy do not admit arbitrages. This is a prerequisite for comparing it with FR and EU economies, since in those economies arbitrages are not possible.

To simplify the analysis let me consider normalized quantities: $p_{t}$ is the normalized price of asset 1 at time $t$ while $w_{t}$ is the normalized wealth of agent 1 at time $t$. They read

$$
\begin{gathered}
p_{t}=\frac{P_{1, t}}{P_{1, t}+P_{2, t}}=\frac{P_{1, t}(1-\beta)}{\beta Y}=\pi^{1} w_{t}+\pi^{2}\left(1-w_{t}\right), \\
w_{t}=\frac{W_{t}^{1}}{W_{t}^{1}+W_{t}^{2}}=\frac{W_{t}^{1}(1-\beta)}{Y}= \\
=w_{t-1}\left(\frac{\beta p_{t}+(1-\beta) s_{t}}{p_{t-1}} \pi^{1}+\frac{1-\beta p_{t}-(1-\beta) s_{t}}{1-p_{t-1}}\left(1-\pi^{1}\right)\right) .
\end{gathered}
$$

Substituting (14) in (13) one can derive a well-defined process for the normalized price of asset $1 .{ }^{10}$ It reads

$$
p_{t}=g\left(p_{t-1}, s_{t}\right)=p_{t-1}+\frac{(1-\beta)\left(\pi^{1}-p_{t-1}\right)\left(p_{t-1}-\pi^{2}\right)\left(s_{t}-p_{t-1}\right)}{p_{t-1}\left(1-p_{t-1}\right)-\beta\left(\pi^{1}-p_{t-1}\right)\left(p_{t-1}-\pi^{2}\right)} .
$$

Proposition 4 of Bottazzi et al. (2017) shows that, in the long-run, only one among three possible cases occurs. Since such result will be crucial for the analysis, I restate their Proposition in terms of the quantities introduced here.

Proposition 4.2 (Bottazzi-Dindo-Giachini). In the SGK economy defined in advance, under assumptions $\mathbf{A} \mathbf{1}$ - A3 one of following occurs

i) if $\mu\left(\pi^{2}, \pi^{1}\right)<0$ then agent 1 dominates and agent 2 vanishes,

ii) if $\mu\left(\pi^{1}, \pi^{2}\right)<0$ then agent 2 dominates and agent 1 vanishes,

iii) if $\mu\left(\pi^{2}, \pi^{1}\right)>0$ and $\mu\left(\pi^{1}, \pi^{2}\right)>0$ then both agents survive and

$$
\operatorname{Prob}\left\{\liminf _{t \rightarrow \infty} p_{t}=\pi^{2} \text { and } \limsup _{t \rightarrow \infty} p_{t}=\pi^{1}\right\}=1 .
$$

\footnotetext{
${ }^{10}$ Equation (15) has been derived for the first time by Bottazzi et al. (2018) to study momentum and reversal. The only difference with respect to the framework considered here is that they model uncertainty in terms of a Markov process.
} 
Proof. See Appendix B.

Building upon such result it is possible to prove the following Proposition.

Proposition 4.3. In the economies defined in advance under assumptions A1 A3

1- if beliefs are such that either case i) or case ii) of Proposition 4.2 holds, then

$$
\lim _{t \rightarrow \infty} p_{t}=\lim _{t \rightarrow \infty} p_{t}^{E U} \quad \text { a.s. }
$$

2- if beliefs are such that case iii) of Proposition 4.2 holds, then, calling agent $i$ the one with the most accurate beliefs, for $t \rightarrow \infty$ it is

$$
K L\left(p_{t}^{F R} \| p_{t}^{E U}\right)=K L\left(\pi^{*} \| \pi^{i}\right)>K L\left(\pi^{*} \| \mathrm{E}\left[p_{t}\right]\right)=K L\left(p_{t}^{F R} \| \mathrm{E}\left[p_{t}\right]\right)
$$

Proof. See Appendix C.

Proposition 4.3 shows that the long-run pricing performance of a SGK economy either matches the one obtained in a EU economy, or it is such that expected normalized prices are "closer" to FR values than those achieved in the long-run by the EU economy. The latter occurs when agents' beliefs allow to more than one agent to survive. According to Corollary 4 and Proposition 5 of Bottazzi et al. (2017), this is a possible and, indeed, generic outcome of market selection in SGK economies. Notice, however, that the inequality in point 2 of Proposition 4.3 involves relative entropy, which is not a proper distance ${ }^{11}$ and it is defined for probability distributions. Thus, the result should be understood in the following way. Consider that in both FR and EU economies, long-run normalized prices show the most accurate beliefs in the market. Hence normalized prices, in the long-run, provide the best evaluation, among agents' ones, of assets' likelihood to pay the dividend. ${ }^{12}$ In a SGK economy, when beliefs are such that both agents survive, normalized prices do not settle down to the evaluations of a single individual, but fluctuate persistently. However, adapting the aggregation argument of Rubinstein (1974), one can say that the market behaves as an "aggregated" agent that invests according to SGK rule and has time-dependent beliefs $\left(p_{t}, 1-p_{t}\right)$. Thus, one can argue that also in a SGK economy long-run normalized prices evaluate assets' likelihood to pay dividends. Under this interpretation, point 2 of Proposition 4.3

\footnotetext{
${ }^{11}$ It is not symmetric and does not respect the triangular inequality.

${ }^{12}$ This can be put into relationship with the idea of market beliefs adopted by Dindo (2015); Massari (2017b); Dindo and Massari (2017).
} 
states that the expected evaluation provided by a SGK economy is more accurate (or approximates better the FR evaluation) than the EU one.

In case $\mathrm{iii}$ ) of Proposition 4.2 one can go further and provide an approximation of $p_{t}$ invariant density using the method proposed by Bottazzi and Giachini (2017). It is based on the diffusive approximation of (15) and, first of all, one should notice that such technique is defined for continuous precesses while the price process is in discrete time. However, as Bottazzi and Giachini (2017) argue, one can obtain a continuous time version of it using an homogeneous Poisson process. Following their passages, I assume that in a certain period of time $d t$ (infinitesimal) a period of the discrete time model can occur with probability $\lambda d t$ while with probability $1-$ $\lambda d t$ nothing happens. Thus I can write down the Chapman-Kolmogorov equation for such process

$$
f_{p}(x, t+d t)=(1-\lambda d t) f_{p}(x, t)+\lambda d t \int d y \int d \pi(s) f_{p}(y, t) \boldsymbol{\delta}(x-g(y, s)),
$$

where $\pi(s)$ is the probability measure of the Bernoulli process which drives the occurrence of states of the world and $\boldsymbol{\delta}(\cdot)$ is the Dirac delta. From (16) one can recover the relative master equation

$$
\frac{\partial f_{p}(x, t)}{\partial t}=\lambda \int d y f_{p}(y, t) \mathbb{K}(x, y)
$$

with

$$
\mathbb{K}(x, y)=\int d \pi(s) \boldsymbol{\delta}(x-g(y, s))-\boldsymbol{\delta}(x-y) .
$$

Now I can take its Kramers-Moyal expansion

$$
\frac{\partial f_{p}(x, t)}{\partial t}=\lambda \sum_{n=1}^{\infty}\left(-\frac{\partial}{\partial x}\right)^{n} \mathcal{D}_{n}(x) f_{p}(x, t),
$$

where

$\mathcal{D}_{n}(x)=\frac{1}{n !} \int d p(p-x)^{n} \mathbb{K}(p, x)=\frac{(1-\beta)^{n}}{n !} \int d \pi(s)\left(\frac{\left(\pi^{1}-x\right)\left(x-\pi^{2}\right)(s-x)}{x(1-x)-\beta\left(\pi^{1}-x\right)\left(x-\pi^{2}\right)}\right)^{n}$.

The Fokker-Planck equation is obtained truncating (17) at the second term

$$
\frac{\partial f_{p}(x, t)}{\partial t}=-\frac{\partial}{\partial x}\left(\lambda \mathcal{D}_{1}(x) f_{p}(x, t)-\frac{\partial}{\partial x}\left(\lambda \mathcal{D}_{2}(x) f_{p}(x, t)\right)\right) .
$$

From the Fokker-Planck equation one can compute the diffusive approximation of $p_{t}$ invariant density setting $\partial f_{p}(x, t) / \partial t=0$ and assuming a vanishing probability 
current at the boundaries. Solving the resulting differential equation yields

$$
f_{p}(x)=\frac{f_{0}}{\mathcal{D}_{2}(x)} \exp \left(\int_{x_{0}}^{x} d y \frac{\mathcal{D}_{1}(y)}{\mathcal{D}_{2}(y)}\right)=f_{0} G(x) \exp \left\{\frac{1}{1-\beta} \int_{\pi^{*}}^{x} d y M(y)\right\}
$$

where $f_{0}$ is a normalization constant,

$$
G(x)=\frac{2\left(\pi^{*}\left(1-\pi^{*}\right)+\left(\pi^{*}-x\right)^{2}\right)^{\frac{2 \beta-1}{1-\beta}}\left(x(1-x)-\left(\pi^{1}-x\right)\left(x-\pi^{2}\right) \beta\right)^{2}}{(1-\beta)^{2}\left(\pi^{1}-x\right)^{2}\left(x-\pi^{2}\right)^{2}}
$$

and

$$
M(x)=\frac{x(1-x)\left(\pi^{*}-x\right)}{\left(\pi^{1}-x\right)\left(x-\pi^{2}\right)\left(\pi^{*}\left(1-\pi^{*}\right)+\left(\pi^{*}-x\right)^{2}\right)} .
$$

The function $M(x)$ is such that $M(x)>0$ if $x<\pi^{*}$ and $M(x)<0$ if $x>\pi^{*}$, hence the exponent is always negative and the global maximum is in $x=\pi^{*}$. Using the asymptotic expansion of the Laplace-like integrals one has

$$
\lim _{\beta \rightarrow 1} \int d x f_{p}(x) x^{n} \sim\left(\pi^{*}\right)^{n} f_{0} G\left(\pi^{*}\right) \sqrt{\frac{2 \pi(1-\beta)}{\left|M^{\prime}\left(\pi^{*}\right)\right|}} .
$$

From the normalization condition one gets

$$
\lim _{\beta \rightarrow 1} \int d x f_{p}(x) x^{n} \sim\left(\pi^{*}\right)^{n}
$$

which means that $\lim _{\beta \rightarrow 1} f_{p}(x)=\boldsymbol{\delta}\left(x-\pi^{*}\right)$. So, provided a $\beta$ close enough to one, when the normalized price converges in distribution to its invariant density it fluctuates in a very narrow interval around $\pi^{*}$, that is, the value $p_{t}^{F R}$ attains. Notice also how for $\beta \rightarrow 1$ the terms after the second in (17) become irrelevant, hence (19) increases its precision as $\beta$ approaches one and, in the limit, it is exact.

Solving the integral in (19) it is possible to derive the analytical form of $f_{p}(x)$ which reads

$$
\begin{aligned}
& f_{p}(x)= \\
& \frac{2 f_{0}\left(x-\pi^{2}\right)^{\frac{2 A_{2}}{1-\beta}-2}\left(\pi^{*}\left(1-\pi^{*}\right)+\left(\pi^{*}-x\right)^{2}\right)^{\frac{2 \beta-2+A_{1}-A_{2}}{1-\beta}}\left(x(1-x)-\left(\pi^{1}-x\right)\left(x-\pi^{2}\right) \beta\right)^{2}}{(1-\beta)^{2}\left(\pi^{1}-x\right)^{\frac{2 A_{1}}{1-\beta}+2}} \\
& \quad \exp \left\{2\left(\frac{A_{1}}{\pi^{1}-\pi^{*}}+\frac{A_{2}}{\pi^{*}-\pi^{2}}\right) \frac{\sqrt{\pi^{*}\left(1-\pi^{*}\right)}}{1-\beta} \arctan \left(\frac{\pi^{*}-x}{\sqrt{\pi^{*}\left(1-\pi^{*}\right)}}\right)\right\},
\end{aligned}
$$


with

$$
A_{i}=\frac{\left(\pi^{*}-\pi^{i}\right)\left(1-\pi^{i}\right) \pi^{i}}{\left(\pi^{1}-\pi^{2}\right)\left(\pi^{*}\left(1-\pi^{*}\right)+\left(\pi^{*}-\pi^{i}\right)^{2}\right)}, \quad i=1,2
$$

In order to provide a graphical example of the approximated density and to evaluate its goodness, in Figure 1 I plot together (21) and the empirical density of $p_{t}$ (found via numerical simulations) for different parameterizations.

Comparing the plots in Figure 1 it is clear how increasing $\beta$ the densities squeeze around $\pi^{*}$. The plots also show how (21) approximates very well the distributions found by numerical simulations, especially when $\beta$ approaches one.

\subsection{Discussion}

The picture that emerges from the analysis is the following: if agents cannot know the true probability according to which states of the world appear, then a SGK economy provides either the same pricing performance of a EU economy or a superior one. That is, decreasing agents' rationality one somehow improves pricing. To understand how this happens, consider the way in which EU agents behave. Following the argument of Sandroni (2000), in equilibrium agents trade in such a way that they end up allocating relatively more wealth to those future events they believe to be more likely. Since beliefs are biased and heterogeneous, the most accurate agent allocates more wealth than the other on the paths that are actually realized, thus, in the long-run, she dominates (Dindo, 2015). In other words the bad trading choices that an agent makes trying to be rational under her very inaccurate beliefs are systematically exploited by another agent with more accurate beliefs. Notice that this holds even when the best beliefs are only slightly better than the worst ones. Thus, in the long-run, prices may end up far away from the FR pricing.

What happens in a SGK economy is rather different. As Bottazzi et al. (2017) argue, agents in such economy trade as if they were expected utility maximizers (with log preferences) under different beliefs than their own. Bottazzi et al. (2017) call them effective beliefs and they turn out to be a price (or wealth) dependent combination of the agent's own beliefs with those of the other agent. The analytical form of agent $i$ 's effective beliefs at time $t,\left(\hat{\pi}_{t}^{i}, 1-\hat{\pi}_{t}^{i}\right)$, can be found following the procedure in Section 5.1 of Bottazzi et al. (2017) (see Appendix D for further details). It yields

$$
\hat{\pi}_{t}^{i}=\left(\beta-\beta \eta\left(p_{t}\right)\right) p_{t}+\left(1-\beta+\beta \eta\left(p_{t}\right)\right) \pi^{i}
$$

with

$$
\eta\left(p_{t}\right)=\frac{(1-\beta)\left(\pi^{1}-p_{t}\right)\left(p_{t-1}-\pi^{2}\right)}{p_{t}\left(1-p_{t}\right)-\beta\left(\pi^{1}-p_{t}\right)\left(p_{t}-\pi^{2}\right)} .
$$


Notice how when agent $i$ 's wealth share is close to one then one has $\hat{\pi}_{t}^{i}=\pi^{i}$, while, when her wealth share is close to zero, it is $\hat{\pi}_{t}^{i}=\beta \pi^{j}+(1-\beta) \pi^{i}$, with $j$ indicating the other agent. Thus, when $\pi^{1}, \pi^{2}, \beta$, and $\pi^{*}$ are such that either case i) or $i$ ) of Proposition 4.2 occurs, the agent with the most accurate beliefs has also the most accurate effective beliefs on average, meaning that her allocation of wealth to future events is better than the one of the other agent. When, instead, case iii) of Proposition 4.2 occurs, agents' effective beliefs are on average equally accurate. Notice that this happens only if $\pi^{2}<\pi^{*}<\pi^{1}{ }^{13}$ hence effective beliefs, combining agents' own beliefs, are able to let agents allocate wealth to future events in a way that is more in line with the true probability of observing paths. In other words, they allocate wealth in a less extreme manner than how they would have done being rational under their own beliefs. This basically means that using a simple rule instead of maximizing preferences may prevent from taking bad decisions driven by wrong beliefs. As Bottazzi et al. (2017) put it, non-optimality of agents' behavior may correct for non-optimality of beliefs. This improvement in decision making at the individual level is reflected at the aggregate level, allowing to enhance pricing performances.

Effective beliefs also highlight the role of $\beta$ in improving pricing: as $\beta$ approaches one agents behave as if they were increasingly accurate since the degree of belief correction applied becomes stronger. ${ }^{14}$ Moreover, the fact that in the limit of $\beta \rightarrow 1$ the prices of a SGK economy characterized by long-run survival of both agents converge to those of a FR economy offers the opportunity to make more general considerations. Indeed it proves that if an economic system shows some sort of rationality at the aggregate level it does not imply that all the agents that compose such system have to be rational in the sense economists are accustomed to. However one should not mistakenly believe that it is possible to reverse the argument and justify a rational expectation approach to describe the price dynamics coming out from a system in which agents with heterogeneous beliefs use simple rules. That is, even if in the limit of agents investing all of their wealth prices of one model converge to the ones of the other, for a $\beta$ very close to 1 the SGK economy prices still fluctuates in a way in which the rational expectation approach cannot deal with.

\section{Conclusion}

in this paper I study the link between agents' rationality and pricing in economies characterized by uncertainty and heterogeneity. To do that I use as benchmark the

\footnotetext{
${ }^{13}$ From (9), by means of Jensen's inequality and the properties of relative entropy, one can easily show that $\pi^{2}<\pi^{1}<\pi^{*}$ implies $\mu\left(\pi^{2}, \pi^{1}\right)<0$ while $\pi^{*}<\pi^{2}<\pi^{1}$ implies $\mu\left(\pi^{1}, \pi^{2}\right)<0$.

${ }^{14}$ See Figure 1 of Bottazzi et al. (2017) for a graphical representation.
} 
pricing of an economy where agents are fully rational, and I compare the lung-run pricing outcomes of two economies where agents have heterogeneous and incorrect beliefs. In one economy agents make their decisions maximizing their preferences under their beliefs, while in the other they behave accordingly to the Subjective Generalized Kelly rule. What I find is that prices of the Subjective Generalized Kelly economy are more in line with the fully rational pricing than those of the utility maximizers economy. This result is driven by the fact that non-optimal decision rules may correct for non-correct beliefs. Moreover I find that, in the limit where agents' discount factor goes to 1, prices of the Subjective Generalized Kelly economy converge to those of the fully rational economy. My conclusions go in the direction outlined by Gigerenzer and Brighton (2009): when a perfect knowledge of the world is not possible, solving complicated problems to decide how to behave makes little sense and fast-and-frugal decision rules may improve the overall outcome.

\section{Acknowledgments}

This research has received funding from the European Union's Horizon 2020 research and innovation programme under grant agreement No 640772 - DOLFINS. I am deeply indebited to Giulio Bottazzi, Pietro Dindo, Giovanni Dosi, Alan Kirman, Filippo Massari and Andrea Roventini for all the precious lessons, comments and advices they gave me. I would also thank Francesco Lamperti, Matteo Sostero, Mattia Guerini and all my collegues at the Institute of Economics of Sant'Anna. Special thanks go to Giulia Rossello for supporting me during the development of this paper and for the discussions concerning the ideas put forward here. I retain the paternity of all the errors that remain.

\section{References}

Alchian, A. (1950). Uncertainty, evolution, and economic theory. Journal of Political Economy 58, 211-221.

Alfarano, S., T. Lux, and F. Wagner (2008). Time variation of higher moments in a financial market with heterogeneous agents: An analytical approach. Journal of Economic Dynamics and Control 32(1), 101-136.

Anufriev, M. and G. Bottazzi (2010). Market equilibria under procedural rationality. Journal of Mathematical Economics 46, 1140-1172.

Anufriev, M. and P. Dindo (2010). Wealth-driven selection in a financial market 
with heterogeneous agents. Journal of Economic Behavior and Organization 73, $327-358$.

Anufriev, M. and C. Hommes (2012). Evolutionary selection of individual expectations and aggregate outcomes in asset pricing experiments. American Economic Journal: Microeconomics 4(4), 35-64.

Arthur, W., J. Holland, B. LeBaron, R. Palmer, and P. Tayler (1997). Asset pricing under endogenous expectations in an artificial stock market. In W. Arthur, S. Durlauf, and D. Lane (Eds.), The Economy as an Evolving Complex System II, pp. 15-44. Addison-Wesley.

Blume, L. and D. Easley (2006). If you are so smart why aren't you rich? Belief selection in complete and incomplete markets. Econometrica 74, 929-966.

Blume, L. and D. Easley (2009). The market organism: Long-run survival in markets with heterogenous traders. Journal of Economic Dynamics and Control 33, 1023-1035.

Bottazzi, G. and P. Dindo (2015, September). Drift criteria for persistence of discrete stochastic processes on the line. LEM Papers Series 2015/26, Laboratory of Economics and Management (LEM), Sant'Anna School of Advanced Studies, Pisa, Italy.

Bottazzi, G., P. Dindo, and D. Giachini (2017). Long-run heterogeneity in an exchange economy with fixed-mix traders. Economic Theory.

Bottazzi, G., P. Dindo, and D. Giachini (2018). Momentum and reversal in financial markets with persistent heterogeneity. LEM Papers Series 2018/04, Laboratory of Economics and Management (LEM), Sant'Anna School of Advanced Studies, Pisa, Italy.

Bottazzi, G. and D. Giachini (2016). Far from the madding crowd: Collective wisdom in prediction markets. LEM Papers Series 2016/14, Laboratory of Economics and Management (LEM), Sant'Anna School of Advanced Studies, Pisa, Italy.

Bottazzi, G. and D. Giachini (2017). Wealth and price distribution by diffusive pproximation in a repeated prediction market. Physica A: Statistical Mechanics and its Applications 471, 473-479.

Breiman, L. (1961). Optimal gambling systems for favorable games. Proceedings of the 4th Berkley Symposium on Mathematical Statistics and Probability 1, 63-68. 
Brock, W. and C. Hommes (1997). A rational route to randomness. Econometrica 65, 1059-1095.

Brock, W. and C. Hommes (1998). Heterogeneous beliefs and routes to chaos in a simple asset pricing model. Journal of Economic Dynamics and Control 22, $1235-1274$.

Bullard, J. and J. Duffy (1999). Using genetic algorithms to model the evolution of heterogeneous beliefs. Computational Economics 13(1), 41-60.

Camerer, C. F., G. Loewenstein, and M. Rabin (2011). Advances in behavioral economics. Princeton University Press.

Chiarella, C., R. Dieci, and L. Gardini (2002). Speculative behaviour and complex asset price dynamics: a global analysis. Journal of Economic Dynamics and Control 49, 173-197.

Chiarella, C., R. Dieci, and L. Gardini (2006). Asset price and wealth dynamics in a financial market with heterogeneous agents. Journal of Economic Dynamics and Control 30 (9-10), 1755-1786.

Chiarella, C., R. Dieci, and X.-Z. He (2009). Heterogeneity, market dynamics, and asset price dynamics. In T. Hens and K. Schenk-Hoppé (Eds.), Handbook of Financial Markets: Dynamics and Evolution. North-Holland (Handbooks in Economics Series).

Chiarella, C. and X. He (2001). Asset price and wealth dynamics under heterogeneous expectations. Quantitative Finance 1, 509-526.

Cvitanić, J., E. Jouini, S. Malamud, and C. Napp (2012). Financial markets equilibrium with heterogeneous agents. Review of Finance 16(1), 285-321.

Day, R. and W. Huang (1990). Bulls, bears and market sheep. Journal of Economic Behaviour and Organization 14, 299-329.

Dindo, P. (2015). Survival in speculative markets. LEM Papers Series 2015/32, Laboratory of Economics and Management (LEM), Sant'Anna School of Advanced Studies, Pisa, Italy.

Dindo, P. and F. Massari (2017). The wisdom of the crowd in dynamic economies. Working Paper Series 17/17, Department of Economics, University of Venice "Ca' Foscari". 
Dosi, G., M. Napoletano, A. Roventini, J. E. Stiglitz, and T. Treibich (2017). Rational heuristics? expectations and behaviors in evolving economies with heterogeneous interacting agents. LEM Papers Series 2017/31, Laboratory of Economics and Management (LEM), Sant'Anna School of Advanced Studies, Pisa, Italy.

Fehr, E. and J.-R. Tyran (2005). Individual irrationality and aggregate outcomes. The Journal of Economic Perspectives 19(4), 43-66.

Franke, R. and R. Sethi (1998). Cautious trend-seeking and complex asset price dynamics. Research in Economics 52, 61-79.

Friedman, M. (1953). Essays in Positive Economics. Univ. Chicago Press.

Gallegati, M., G. Giulioni, A. Kirman, and A. Palestrini (2011). Whats that got to do with the price of fish? buyers behavior on the ancona fish market. Journal of Economic Behavior \& Organization 80(1), 20-33.

Gigerenzer, G. and H. Brighton (2009). Homo heuristicus: Why biased minds make better inferences. Topics in Cognitive Science 1(1), 107-143.

Gigerenzer, G. and W. Gaissmaier (2011). Heuristic decision making. Annual review of psychology 62, 451-482.

Gigerenzer, G., P. M. Todd, t. ABC Research Group, et al. (1999). Simple heuristics that make us smart. Oxford University Press.

Gode, D. K. and S. Sunder (1993). Allocative efficiency of markets with zerointelligence traders: Market as a partial substitute for individual rationality. Journal of political economy 101(1), 119-137.

Härdle, W. and A. Kirman (1995). Nonclassical demand: A model-free examination of price-quantity relations in the marseille fish market. Journal of Econometrics 67(1), 227-257.

Hommes, C. (2006). Heterogeneous agent models in economics and finance. In K. Judd and L. Tesfatsion (Eds.), Handbook of Computational Economics Vol. 2: Agent-Based Computational Economics. North-Holland (Handbooks in Economics Series), Amsterdam.

Iori, G. (2002). A microsimulation of traders activity in the stock market: the role of heterogeneity, agents' intercations and trade frictions. Journal of Economic Dynamics and Control 49, 269-285. 
Jouini, E. and C. Napp (2011). Unbiased disagreement in financial markets, waves of pessimism and the risk-return trade-off. Review of Finance 15(3), 575-601.

Kets, W., D. M. Pennock, R. Sethi, and N. Shah (2014). Betting strategies, market selection, and the wisdom of crowds. In Twenty-Eighth AAAI Conference on Artificial Intelligence.

Kirman, A. (1991). Epidemics of opinion and speculative bubbles in financial markets. In M. Taylor (Ed.), Money and Financial Markets, Chapter 17, pp. 354-368. Blackwell, Cambridge.

Kirman, A. (1993). Ants, rationality and recruitment. Quarterly Journal of Economics 108, 137-156.

Kirman, A. (2010). Complex economics: individual and collective rationality. Routledge.

Kirman, A. P. and N. J. Vriend (2000). Learning to be loyal. a study of the marseille fish market. Interaction and Market structure, 33-56.

Knight, F. H. (1921). Risk, uncertainty and profit. New York: Hart, Schaffner and Marx.

Kogan, L., S. A. Ross, J. Wang, and M. M. Westerfield (2006). The price impact and survival of irrational traders. The Journal of Finance 61(1), 195-229.

Kogan, L., S. A. Ross, J. Wang, and M. M. Westerfield (2017). Market selection. Journal of Economic Theory 168, 209-236.

Lucas, R. E. (1978). Asset prices in an exchange economy. Econometrica 46(6), $1429-1445$.

Lux, T. (1995). Herd behaviour, bubbles and crashes. The Economic Journal 105, 881-896.

Lux, T. and M. Marchesi (1999). Scaling and criticality in a stochastic multi-agent model of financial market. Nature 397, 498-500.

Massari, F. (2017a). Market selection in large economies: A matter of luck. SSRN 2559468.

Massari, F. (2017b). Markets with heterogeneous beliefs: A necessary and sufficient condition for a trader to vanish. Journal of Economic Dynamics and Control 78, 190-205. 
Rubinstein, M. (1974). An aggregation theorem for securities markets. Journal of Financial Economics 1(3), 225-244.

Sandroni, A. (2000). Do markets favor agents able to make accurate predictions. Econometrica 68(6), 1303-1341.

Sethi, R. (1996). Endogenous regime switching in speculative market. Structural Change and Economic Dynamics 7, 99-118.

Simon, H. (1955). A behavioral model of rational choice. The Quarterly Journal of Economics 69(1), 99-118.

Tedeschi, G., G. Iori, and M. Gallegati (2012). Herding effects in order driven markets: The rise and fall of gurus. Journal of Economic Behavior \& Organization $81(1), 82-96$.

Tversky, A. and D. Kahneman (1974). Judgment under uncertainty: Heuristics and biases. Science 185, 1124-1131.

\section{A Proof of Proposition 4.1}

Here I follow Blume and Easley (2009) to show how normalized prices (and not only state prices) reveal the beliefs of the most accurate agent. Let me define $q_{t}^{E U, s}$ as the state price of the state claim paying in state $s$ after the partial history $\sigma^{t}$, $D_{k, t+1}^{s}$ as the dividend that asset $k$ pays in $t+1$ if state $s$ occurs after partial history $\sigma^{t}$ and $P_{k, t+1}^{E U, s}$ as the price of asset $k$ in $t+1$ if state $s$ occurs after partial history $\sigma^{t}$. Then it holds

$$
P_{k, t}^{E U}=\sum_{s=0}^{1} q_{t}^{E U, s}\left(D_{k, t+1}^{s}+P_{k, t+1}^{E U, s}\right), \quad \forall t, k=1,2 .
$$

Since the market is dynamically complete, the agent's optimal consumption problem can be solved using time-zero trading. That is, agents can exchange all the state claims that pay 1 unit of consumption good after history $\sigma^{t}$ and zero otherwise. Call $q^{E U}\left(\sigma^{t}\right)$ the price of such security and $\pi^{i}\left(\sigma^{t}\right)$ the probability agent $i$ assigns to history $\sigma^{t}$ on the basis of her beliefs. From agent $i$ 's first order conditions

$$
\beta^{t} \pi^{i}\left(\sigma^{t}\right) U^{\prime}\left(C_{t}^{E U, i}\right)=\phi^{i} q^{E U}\left(\sigma^{t}\right) \quad \forall t, \sigma^{t},
$$


(where $\phi^{i}$ is agent $i$ 's Lagrange multiplier) one gets

$$
q_{t}^{E U, 1}=\beta \pi^{i} \frac{U^{\prime}\left(C_{1, t+1}^{E U, i}\right)}{U^{\prime}\left(C_{t}^{E U, i}\right)}, \quad q_{t}^{E U, 0}=\beta\left(1-\pi^{i}\right) \frac{U^{\prime}\left(C_{0, t+1}^{E U, i}\right)}{U^{\prime}\left(C_{t}^{E U, i}\right)},
$$

with $C_{s, t+1}^{E U, i}$ denoting agent $i$ 's consumption at date $t+1$ provided history $\sigma^{t}$ and state $s$ realized at $t+1$. Then, substituting in (23), it is

$P_{k, t}^{E U}=\beta\left(\pi^{i} \frac{U^{\prime}\left(C_{1, t+1}^{E U, i}\right)}{U^{\prime}\left(C_{t}^{E U, i}\right)}\left(D_{k, t+1}^{1}+P_{k, t+1}^{E U, 1}\right)+\left(1-\pi^{i}\right) \frac{U^{\prime}\left(C_{0, t+1}^{E U, i}\right)}{U^{\prime}\left(C_{t}^{E U, i}\right)}\left(D_{k, t+1}^{0}+P_{k, t+1}^{E U, 0}\right)\right)$.

Notice that, by continuity, if $C_{t}^{E U, i} \rightarrow Y$ then $P_{1, t}^{E U} \rightarrow \pi^{i} Y \beta /(1-\beta)$ and $P_{2, t}^{E U} \rightarrow$ $\left(1-\pi^{i}\right) Y \beta /(1-\beta)$. Following the method presented in Sandroni (2000), Blume and Easley (2006), Blume and Easley (2009), one can take agents' first order conditions, iteratively substitute, and, by the strong law of large numbers, one has $\lim _{t \rightarrow \infty} \frac{1}{t} \log \frac{U^{\prime}\left(C_{t}^{E U, i}\right)}{U^{\prime}\left(C_{t}^{E U, j}\right)}=\pi^{*} \log \frac{\pi^{j}}{\pi^{i}}+\left(1-\pi^{*}\right) \log \frac{1-\pi^{j}}{1-\pi^{i}}=K L\left(\pi^{*} \| \pi^{i}\right)-K L\left(\pi^{*} \| \pi^{j}\right)<0$,

with $i$ denoting the most accurate agent and $j$ the other one. Thus, by the Inada conditions one has $C_{t}^{E U, j} \rightarrow 0$ which implies $C_{t}^{E U, i} \rightarrow Y$. Considering the normalized prices one gets the statement.

\section{B Proof of Proposition 4.2}

Under A1 there exists a $\varepsilon>0$ such that $\varepsilon \leq \pi^{2}<\pi^{2} \leq 1-\varepsilon$. Define the process

$$
z_{t}=\log \frac{w_{t}}{1-w_{t}}=\log \frac{p_{t}-\pi^{2}}{\pi^{1}-p_{t}}
$$

and let $\gamma\left(p_{t-1}, s_{t}\right)=z_{t}-z_{t-1}$. Considering (15) one can show that for $p \in\left[\pi^{2}, \pi^{1}\right]$ it is

$$
0<\gamma(p, 1)<\log \frac{1-\varepsilon}{\varepsilon}
$$

and

$$
\log \frac{\varepsilon}{1-\varepsilon}<\gamma(p, 0)<0
$$

Given the continuity of $\gamma(p, s)$ in $p$, the two inequalities imply that the process $\left\{z_{t}\right\}$ has bounded increments with both positive and negative finite increments as 
defined in Bottazzi and Dindo (2015). Consider now $\mathcal{M}(z)=\mathrm{E}\left[z_{t+1}-z_{t} \mid z_{t}=z\right]$ and notice that

$$
\begin{aligned}
& \lim _{z \rightarrow+\infty} \mathcal{M}(z)=-\mu\left(\pi^{2}, \pi^{1}\right), \\
& \lim _{z \rightarrow-\infty} \mathcal{M}(z)=\mu\left(\pi^{1}, \pi^{2}\right) .
\end{aligned}
$$

Moreover, it follows from Lemma 4 of Bottazzi et al. (2017) that

$$
\mu\left(\pi^{1}, \pi^{2}\right)>-\mu\left(\pi^{2}, \pi^{1}\right) .
$$

Thus, by continuity, there exist a $m>0$ and a $\epsilon>0$ such that, under the condition in $i$ ), for all $z>m$ and $z<-m$ it is $\mathcal{M}(z)>\epsilon$. By Theorem 3.1 of Bottazzi and Dindo (2015) this implies $\lim _{t \rightarrow \infty} z_{t}=+\infty$ a.s., which delivers the statement in $i$ ).

If the condition in $i i$ ) is satisfied then, by continuity, there exist a $m>0$ and a $\epsilon>0$ such that for all $z>m$ and $z<-m$ it is $\mathcal{M}(z)<-\epsilon$. By Corollary 3.1 of Bottazzi and Dindo (2015) this implies $\lim _{t \rightarrow \infty} z_{t}=-\infty$ a.s., which delivers the statement in $i i)$.

If the conditions in $i i i$ ) are satisfied then, by continuity, there exist a $m>0$ and a $\epsilon>0$ such that for all $z>m$ it is $\mathcal{M}(z)<-\epsilon$ and for all $z<-m$ it is $\mathcal{M}(z)>\epsilon$. By Theorem 2.2 of Bottazzi and Dindo (2015) this implies $\lim \sup _{t \rightarrow \infty} z_{t}=+\infty$ and $\liminf _{t \rightarrow \infty} z_{t}=-\infty$ a.s., which deliver the statements in $\left.i i i\right)$.

\section{Proof of Proposition 4.3}

The first point follows from Proposition 4.2 and Corollary 3 of Bottazzi et al. (2017): Corollary 3 of Bottazzi et al. (2017) proves that the agent with the most accurate beliefs survives, thus if one agent dominates, she cannot be the one with the least accurate beliefs, since this would imply that the agent with most accurate beliefs vanishes. Hence when beliefs are such that either $i$ ) or $i i$ ) holds, in the long-run there are no differences in terms of pricing between an EU economy and an SGK economy. To prove the second point consider equation (15) and call $p^{1}(p)=g(p, 1)$ and $p^{0}(p)=g(p, 0)$. Defining the function

$$
\eta(p)=\frac{(1-\beta)\left(\pi^{1}-p\right)\left(p-\pi^{2}\right)}{p(1-p)-\beta\left(\pi^{1}-p\right)\left(p-\pi^{2}\right)}
$$

one has $p^{1}(p)=p+(1-p) \eta(p), p^{0}(p)=p(1-\eta(p))$, and $0<\eta(p)<1$ for $p \in\left(\pi^{2}, \pi^{1}\right)$.

When both agents survive it is $\mathrm{E}\left[\log w_{t+1}^{i}-\log w_{t}^{i}\right]=0$ for $t$ sufficiently large, 
thus

$$
\begin{aligned}
0= & \mathrm{E}\left[\mathrm{E}\left[\log \frac{w_{t+1}^{i} \mid}{w_{t}^{i}} \mid p_{t}\right]\right]=\mathrm{E}\left[\pi^{*} \log \left(\frac{\beta p^{1}\left(p_{t}\right)+1-\beta}{p_{t}} \pi^{i}+\frac{\beta\left(1-p^{1}\left(p_{t}\right)\right)}{1-p_{t}}\left(1-\pi^{i}\right)\right)+\right. \\
& \left.+\left(1-\pi^{*}\right) \log \left(\frac{\beta p^{0}\left(p_{t}\right)}{p_{t}} \pi^{i}+\frac{\beta\left(1-p^{0}\left(p_{t}\right)\right)+1-\beta}{1-p_{t}}\left(1-\pi^{i}\right)\right)\right] .
\end{aligned}
$$

Let me define the function

$$
\begin{aligned}
\nabla(p)= & \pi^{*} \log \left(\frac{\beta p^{1}(p)+1-\beta}{p} \pi^{i}+\frac{\beta\left(1-p^{1}(p)\right)}{1-p}\left(1-\pi^{i}\right)\right)+ \\
& +\left(1-\pi^{*}\right) \log \left(\frac{\beta p^{0}(p)}{p} \pi^{i}+\frac{\beta\left(1-p^{0}(p)\right)+1-\beta}{1-p}\left(1-\pi^{i}\right)\right)
\end{aligned}
$$

such that, substituting the definition of $p^{1}(p)$ and $p^{0}(p)$ in (25), one gets

$$
\begin{aligned}
\nabla(p)= & \pi^{*} \log \frac{(\beta-\beta \eta(p)) p+(1-\beta+\beta n(p)) \pi^{i}}{p}+ \\
& +\left(1-\pi^{*}\right) \log \frac{1-(\beta-\beta \eta(p)) p-(1-\beta+\beta n(p)) \pi^{i}}{1-p}
\end{aligned}
$$

Now, substituting with the definition of $\eta(p)$, it is

$$
\begin{aligned}
\nabla(p)= & \pi^{*} \log \frac{\left(\beta\left(1-\pi^{1}-\pi^{2}\right)-(1-\beta) \pi^{i}\right) p+\beta \pi^{1} \pi^{2}+(1-b) \pi^{i}}{-(1-\beta) p^{2}+\left(1-\beta \pi^{1}-\beta \pi^{2}\right) p+\beta \pi^{1} \pi^{2}}+ \\
& +\left(1-\pi^{*}\right) \log \frac{\left(\beta\left(1-\pi^{1}-\pi^{2}\right)+(1-\beta)\left(1-\pi^{i}\right)\right) p+\beta \pi^{1} \pi^{2}}{-(1-\beta) p^{2}+\left(1-\beta \pi^{1}-\beta \pi^{2}\right) p+\beta \pi^{1} \pi^{2}}
\end{aligned}
$$

Let me consider the functions

$$
\begin{aligned}
& z_{1}(p)=\left(\beta\left(1-\pi^{1}-\pi^{2}\right)-(1-\beta) \pi^{i}\right) p+\beta \pi^{1} \pi^{2}+(1-\beta) \pi^{i} \\
& z_{2}(p)=\left(\beta\left(1-\pi^{1}-\pi^{2}\right)+(1-\beta)\left(1-\pi^{i}\right)\right) p+\beta \pi^{1} \pi^{2} \\
& z_{3}(p)=-(1-\beta) p^{2}+\left(1-\beta \pi^{1}-\beta \pi^{2}\right) p+\beta \pi^{1} \pi^{2}
\end{aligned}
$$

such that $\nabla(p)=\pi^{*} \log z_{1}(p)+\left(1-\pi^{*}\right) \log z_{2}(p)-\log z_{3}(p)$. Notice that for $p \in\left(\pi^{2}, \pi^{1}\right)$ those functions are positive and, while $z_{1}(p)$ and $z_{2}(p)$ are linear in $p, z_{3}(p)$ is a concave quadratic polynomial. Considering the second derivative of $\nabla(p)$, one has

$$
\frac{\partial^{2} \nabla(p)}{\partial p^{2}}=-\frac{\pi^{*}}{\left(p-x_{1}\right)^{2}}-\frac{1-\pi^{*}}{\left(p-x_{2}\right)^{2}}+\frac{1}{\left(p-x_{3}\right)^{2}}+\frac{1}{\left(x_{4}-p\right)^{2}},
$$


with $x_{1}, x_{2}, x_{3}, x_{4}$ such that $z_{1}\left(x_{1}\right)=0, z_{2}\left(x_{2}\right)=0, z_{3}\left(x_{3}\right)=0$, and $z_{3}\left(x_{4}\right)=0$. Notice that $x_{3}<0<\pi^{2}<\pi^{1}<1<x_{4}$ and if both $x_{1}$ and $x_{2}$ do not belong to the interval $\left(x_{3}, x_{4}\right)$ then $\partial^{2} \nabla(p) / \partial p^{2}>0$. To show that this is the case, consider that $z_{1}(p)=z_{3}(p)$ for $p=\pi^{i}$ and $p=1$, while $z_{2}(p)=z_{3}(p)$ for $p=0$ and $p=\pi^{i}$. Since $z_{3}(0)>0, z_{3}\left(\pi^{2}\right)>0, z_{3}\left(\pi^{1}\right)>0, z_{3}(1)>0$, it cannot be the case that $x_{1}, x_{2} \in\left(x_{3}, x_{4}\right)$.

Thus $\nabla(p)$ is strictly convex for $p \in\left(\pi^{2}, \pi^{1}\right)$ and from equations $(24)-(25)$, by Jensen's inequality, one has

$$
0=\mathrm{E}\left[\nabla\left(p_{t}\right)\right]>\nabla\left(\mathrm{E}\left[p_{t}\right]\right) .
$$

Considering equation (26) and noticing how $0<\beta\left(1-\eta\left(p_{t}\right)\right)<1$ for $p_{t} \in\left(\pi^{2}, \pi^{1}\right)$, by Jensen's inequality it is

$$
\nabla\left(\mathrm{E}\left[p_{t}\right]\right)>\left(1-\beta+\beta \eta\left(\mathrm{E}\left[p_{t}\right]\right)\left(\pi^{*} \log \frac{\pi^{i}}{\mathrm{E}\left[p_{t}\right]}+\left(1-\pi^{*}\right) \log \frac{1-\pi^{i}}{1-\mathrm{E}\left[p_{t}\right]}\right)\right.
$$

which, in turn, implies

$$
K L\left(\pi^{*} \| \pi^{i}\right)>K L\left(\pi^{*} \| \mathrm{E}\left[p_{t}\right]\right)
$$

To get the statement couple such result with Proposition 4.1 and the FR pricing.

\section{Effective Beliefs}

Similarly to what done at the beginning of Appendix C, define $p^{1}\left(p_{t}\right)=g\left(p_{t}, 1\right)=$ $p_{t}+\left(1-p_{t}\right) \eta\left(p_{t}\right)$ and $p^{0}\left(p_{t}\right)=g\left(p_{t}, 0\right)=p_{t}\left(1-\eta\left(p_{t}\right)\right)$. Then, knowing that a log-optimal agent invests in date $t$ contingent claims proportionally to her beliefs, to match the wealth dynamics of a SGK economy, agent $i$ 's effective beliefs must be such that

$$
\left\{\begin{array}{l}
\frac{\hat{\pi}_{t}^{i}}{\hat{p}_{t}}=\frac{\beta p^{1}\left(p_{t}\right)+1-\beta}{p_{t}} \pi^{i}+\frac{\beta\left(1-p^{1}\left(p_{t}\right)\right)}{1-p_{t}}\left(1-\pi^{i}\right), \\
\frac{1-\hat{\pi}_{t}^{i}}{1-\hat{p}_{t}}=\frac{\beta p^{0}\left(p_{t}\right)}{p_{t}} \pi^{i}+\frac{\beta\left(1-p^{0}\left(p_{t}\right)\right)+1-\beta}{1-p_{t}}\left(1-\pi^{i}\right),
\end{array}\right.
$$

with $\hat{p}_{t}$ the date $t$ price of the contingent claim on the realization of state 1 at $t+1$ in the log-optimal economy. Substituting with the definition and solving one of the two equations of the system under the assumption $\hat{p}_{t}=p_{t}$ yields equation (22). To check that $\hat{p}_{t}=p_{t}$ is indeed true when beliefs are as in (22), consider that $\hat{p}_{t}$ is the wealth weighted average of log optimal agents' beliefs, thus

$\hat{p}_{t}=w_{t} \hat{\pi}_{t}^{1}+\left(1-w_{t}\right) \hat{\pi}_{t}^{2}=\left(\beta-\beta \eta\left(p_{t}\right)\right) p_{t}+\left(1-\beta+\beta \eta\left(p_{t}\right)\right)\left(w_{t} \pi^{1}+\left(1-w_{t}\right) \pi^{2}\right)=p_{t}$. 

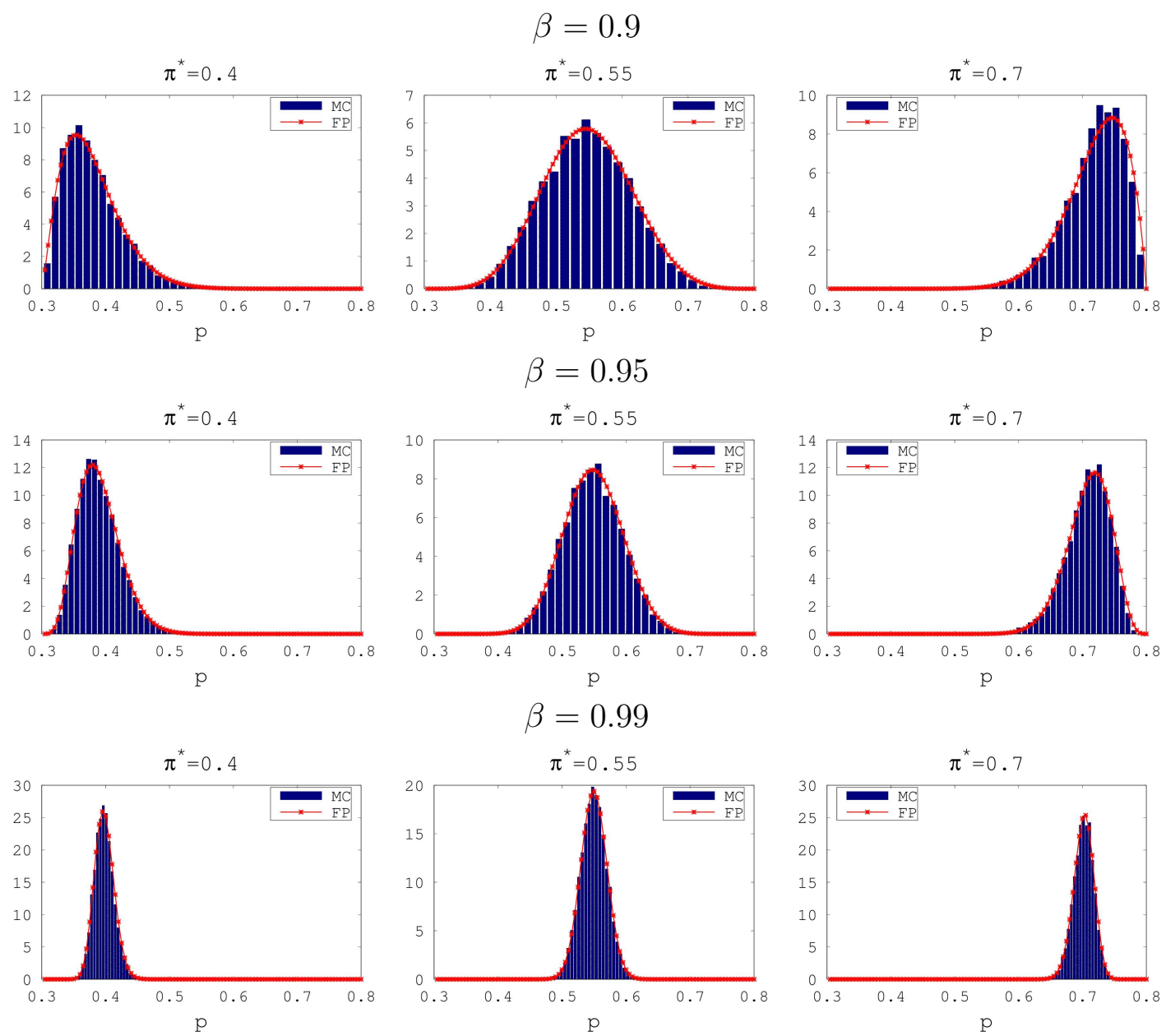

Figure 1: Approximated invariant density distributions (labeled FP in the plots) and empirical densities from simulations (labeled MC in the plots) computed keeping fixed $\pi^{1}=0.8$ and $\pi^{2}=0.3$ while considering different values of $\beta$ and $\pi^{*}$. Details of the simulations: 10000 independent runs of 10000 periods each with starting normalized price $\pi^{*}$. The distribution of $p_{t}$ from the numerical experiment is computed considering the last price of every run. Using different initial conditions does not entail any substantial difference. 